\title{
El rol del profesor desde la perspectiva de los Espacios de Trabajo Matemático
}

\section{The Teacher's role from the perspective of Mathematical Working Spaces}

\author{
Inés $\mathrm{M}^{\mathrm{a}}$ Gómez-Chacón ${ }^{*}$ \\ Alain Kuzniak $^{* * *}$ \\ Laurent Vivier ${ }^{* * *}$
}

\begin{abstract}
Resumen
En este artículo se presenta el modelo Espacio de Trabajo Matemático (ETM). Se pone de manifiesto el dinamismo de este modelo para reflexionar sobre la acción del profesor en el aula y sobre elementos importante a tener en cuenta en su formación. Los ETM se construyen con base en el conocimiento matemático y la experiencia del sujeto al hacer matemáticas, de ahí que su organización remita a dos planos: el epistemológico y el cognitivo, que serán el centro de la discusión de este trabajo. Se presentan las contribuciones al monográfico en las dinámicas que establecen el rol del docente en los conocimientos y las interacciones en el aula, desde dos puntos de vista en la formación de docente y en el rol del docente con los alumnos.
\end{abstract}

Palabras clave: Espacio de Trabajo Matemático. Conocimiento del Profesor. Desarrollo Profesional del Profesor de Matemáticas. Educación Matemática.

\begin{abstract}
In this article, the Mathematical Working Space model (MWS) is presented. It shows the dynamism of this model to reflect on the teacher's actions in the classroom and on the important elements to consider in their training. The MWS is built based on the mathematical knowledge and experience of the subject to do mathematics, hence the organization refers to two planes: the epistemological and the cognitive one, which will be the centre of the discussion in this work. Monographic contributions in the dynamics that are established in the role of the teacher in the knowledge and the interactions in the classroom are presented, from two points of view, from the teacher's training and from the teacher's role with the students.
\end{abstract}

Keywords: Mathematical Working Space. Teacher's knowledge. Mathematics Professional Development. Mathematical Education

\footnotetext{
* Doctora, Universidad Complutense de Madrid (UCM), Facultad de Ciencias Matemáticas, Madrid, España. Dirección postal: Ciudad Universitaria, Plaza de Ciencias 3, 28040 Madrid, España. E-mail: igomezchacon@mat.ucm.es.

* Doctor, LDAR (EA 4434), Universidad de Paris Diderot -Case 7018- Place Aurélie Nemours Bâtiment Sophie Germain 75205 Paris Cedex 13 Francia; alain.kuzniak@univ-paris-diderot.fr.

**** Doctor en matemática de la universidad de Tours, 60 rue du Plat D'Etain 37020 Tours Cedex 1 Francia; Habilitado para Dirigir Investigaciones (HDR) en Didáctica de las Matemáticas de la Universidad Paris Diderot, 5 rue Thomas Mann 75205 Paris cedex 13 Francia; Profesor Asociado en el LDAR, Universidad de Paris Diderot -Case 7018- Place Aurélie Nemours Bâtiment Sophie Germain 75205 Paris Cedex 13 Francia; laurent.vivier@univ-paris-diderot.fr.
} 


\section{Introducción}

La enseñanza de la matemática está siendo fuertemente transformada por el contexto social actual, donde la masificación de la educación se sitúa en el ámbito de una competitividad económica cada vez más fuerte y una demanda creciente en los estudiantes de mejores capacidades y formas cualificadas de trabajo. Los diseñadores buscan, a través de actividades estrechamente ligadas al mundo real, el estímulo del alumnado en la resolución de problemas matemáticos. Al mismo tiempo, la aparición de las nuevas tecnologías ha tenido un fuerte impacto tanto en el contenido como en las discusiones de las formas de enseñar y aprender.

En cualquier caso, el trabajo matemático que producen los estudiantes permanece en el corazón de las transformaciones que tienen lugar en la enseñanza y aprendizaje de la matemática y que lógicamente nos conducen a situarlo como uno de los temas centrales de estudio en la Educación matemática. De hecho, el tema del Trabajo matemático es objeto desde hace más de diez años de investigación en estrecha colaboración entre investigadores de varios países. Muestra de ello destacamos los más recientes simposios $^{1}$ en los que se ha identificado y desarrollado el Modelo de Espacios de Trabajo Matemático.

Este número especial de la Revista Boletim de Educação Matemática (BOLEMA) es el resultado de distintos artículos elaborados para el cuarto simposio Espacio de Trabajo Matemático (ETM), cuyo objeto de estudio es el uso de la noción de Espacio de Trabajo Matemático (ETM) en la didáctica de las matemáticas. En particular queremos mostrar como este modelo permite enriquecer el estudio didáctico del trabajo matemático de alumnado y profesorado y ofrecer instrumentos para el análisis del rol del docente, conocimientos e interacciones en el aula.

En las dos primeras partes de este artículo se presentan la perspectiva sobre el trabajo matemático y las bases del enfoque de los ETM. Seguidamente, se describe la organización temática de las contribuciones, terminando con un análisis del trabajo matemático del profesor en el aula y elementos importante a tener en cuenta en su formación.

\section{Una perspectiva didáctica sobre el trabajo matemático}

\footnotetext{
${ }^{1}$ En 2012 el tercero Simposium ETM 3 en Montreal, Canadá, (http://turing.scedu.umontreal.ca/etm), en 2014 el Cuarto Simposium ETM 4, en San Lorenzo de El Escorial, Madrid, España (http://www.mat.ucm.es/imi/ETM4/index.html) y el próximo Simposio que se celebrará en el 2016 en Florina, Grecia, (http://etm5.web.uowm.gr/es).
} 


\section{1 La actividad del matemático como modelo para el trabajo matemático}

Introducir el concepto de trabajo en el marco de la enseñanza conlleva apostar por una visión de escuela formativa. Qué sentido tendrían las actividades matemáticas si no estuvieran orientadas a un objetivo general formativo más amplio como persona inmersa en la sociedad.

Desde los primeros trabajos en Educación matemática, algunos investigadores consideran al alumno como un matemático en ciernes, erigiendo como un modelo para el estudiante la actividad del matemático experto. Estas reflexiones se han traslado a ambiciosas propuestas en el contexto del debate científico (LEGRAND, 1990). En ellas, el alumno no sólo se considera como un aprendiz que aplica unos ejercicios sino como un investigador que explora el dominio matemático. Pero ¿en qué consiste exactamente esta actividad específica que le hace ser reconocido como matemático? Freudenthal (1971) trata de definirla desde la comunidad de matemáticos:

What is mathematics? Of course you know that mathematics is an activity because you are active mathematicians. It is an activity of solving problems, of looking for problems, but it is also an activity of organizing a subject matter. (FREUDENTHAL, 1971, p. 414).

Define las matemáticas o más bien la acción del matemático como una actividad de resolución y de investigación de problemas - lo que supone definir la matemática en relación a la actividad organizada en un área específica. Desde este punto de vista, el trabajo del matemático resulta de su actividad definida como matemático. La comprensión más contemporánea de este trabajo es sin duda la dada por Thurston en "On proof and progress in mathematics" (1994), artículo en el cuál Hacking (2014) ofrece un análisis y según él su lectura debería ser obligatoria para cualquier persona interesada en la filosofía de las matemáticas (p. 61).

Para Thurston (1994) una dificultad de definir la matemática de forma fácil y directa es su carácter esencialmente recursivo de la actividad matemática. Por ello, propone definir las matemáticas como la disciplina más pequeña que satisface las siguientes condiciones:

- Matemática incluye números enteros y la geometría del plano y de los sólidos;

- Las matemáticas son lo que los matemáticos estudian;

- Los matemáticos son aquellos seres humanos que permiten avanzar en el entendimiento humano de las matemáticas.

Hoy no es posible acceder a este trabajo sin añadir nuevos conceptos. El recorrido del alumno seguirá esta génesis incierta de un pensamiento que se nutre de cada una de las 
experiencias anteriores. Por suerte, su camino estará marcado por partida doble, de un lado por el trabajo previo de todos los matemáticos que lo han hecho posible y comprensible, y de otro el de los profesores que van a acompañar en la construcción de su propio Espacio de Trabajo Matemático.

\section{2 Áreas de conocimiento en matemáticas y paradigmas}

Los objetos y los resultados producidos por el trabajo matemático se dividen en áreas de conocimiento que estructuran la investigación matemática y permiten dar cuenta de la diversidad de la actividad matemática.

La diferenciación de los dominios de conocimiento en matemáticas está relacionada con la naturaleza de los objetos estudiados $\mathrm{y}$, esencialmente, con los fundamentos epistemológicos que marcan estas diferencias. Cada dominio o área de conocimiento matemático está especialmente preocupada por un tipo de preguntas que no son directamente matemáticas: así la geometría elemental está particularmente relacionada con la noción de espacio.

Para ceñirse a los temas más trabajadas en Educación matemática, destacaremos una serie de áreas: Aritmética, Álgebra, Análisis, Geometría, Probabilidad y Estadística. Cada una de estas áreas se conectará a temáticas no matemáticas como contar, la simbolización, la generalización, el espacio, el cambio, el azar, la decisión. Esta lista no agota el tema, pero muestra la complejidad y heterogeneidad de los objetos en juego cuando abordamos el trabajo matemático en general.

Las áreas matemáticas o dominios matemáticos están formados por la agregación y organización de conocimientos y como señala Brousseau (2002), esta organización no se corresponde con lo que necesariamente se implementa en la enseñanza. Cada dominio en matemáticas es objeto de diferentes interpretaciones y depende de la adaptación para ser enseñado en una institución académica concreta. El ejemplo de la Geometría muestra que no es posible utilizar el término de forma univoca. Como término Geometría adquiere significados diferentes que dependen tanto de la evolución de las matemáticas como de las instituciones académicas.

Para dar cabida a esta diversidad Houdement y Kuzniak $(1999,2006)$ han introducido en el ámbito de la didáctica una aproximación mediante paradigmas, próximo al concepto de paradigma desarrollado por Kuhn (1962) en su libro sobre La Estructura de las Revoluciones 
Científicas. Un paradigma designará el conjunto de creencias, técnicas y valores compartidos por un grupo científico. El acceso al paradigma se hará con los textos matemáticos y a través de la resolución de una serie de problemas característicos.

Recordemos que la teoría de Kuhn está basada principalmente en el estudio de la física, si se aplica a las matemáticas no es trivial y ha sido objeto de numerosos debates y polémicas (Gillies 1992). Para Kuhn, los paradigmas pueden coexistir y su relación es necesariamente conflictiva. Un nuevo paradigma tiende a eliminar el viejo mientras que en matemáticas, los paradigmas parecen sobrevivir y continuar existiendo y esto es especialmente cierto en la institución escolar. En el caso de las matemáticas, tenemos una idea menos conflictiva donde los paradigmas pueden coexistir para resolver problemas, centrándose en diferentes entradas. Por ejemplo, es posible en el Análisis matemático distinguir varios sub-paradigmas dentro del paradigma de Análisis Estándar que se impone en el siglo XIX, y cada uno de esto sub-paradigmas participa en el desarrollo del trabajo matemático en esta área.

\subsection{Aspectos epistemológicos y cognitivos: el trabajo y la resolución de problemas matemáticos}

Hasta ahora hemos visto sobre todo el desarrollo del trabajo matemático haciendo hincapié en la coherencia epistemológica necesaria para su desarrollo. Pero la componente humana (o social, compartida por todas las instancias de esa actividad) asume una dimensión cognitiva que debe estar relacionado con la dimensión epistemológica.

La resolución de problemas permite establecer una conexión rápida y eficiente entre estos dos aspectos complementarios del trabajo matemático. Ésta ocupa un lugar esencial en el trabajo de los matemáticos y también en la Educación matemática. Gracias a la resolución de problemas los alumnos pondrán en práctica los conocimientos y las técnicas elegidas por cada paradigma. A partir de la acertada distinción hecha en las Teorías de la Actividad (ver VANDEBROUCK, 2013) entre la tarea y la actividad, sostenemos que este desarrollo dependerá de las tareas previstas a un individuo y las actividades que desarrolla. La observación de las tareas y las actividades producidas permite describir los dos aspectosepistemológico y cognitivo- en el trabajo matemático. Así concebido como evolución de estructuras en las actividades matemáticas, los Espacios de Trabajo Matemático y su estudio deben permitir dar cuenta de cómo un determinado conjunto de tareas y actividades terminan 
por estructurar (o no) un trabajo matemático complejo y rico por parte de profesores y estudiantes.

El estudio de las tareas y de las actividades asume dos enfoques complementarios. En el primero, un estudio detallado de los tipos de tareas con sus conocimientos técnicos y teóricos permite diferenciar la estructuración del campo matemático. El segundo, aborda más la distancia entre lo que se espera del estudiante y lo que realmente realiza. Esto requiere de la observación de las prácticas matemáticas propuestas en el ámbito escolar y propuestas en los ámbitos profesionales y cotidianos, con objeto de la identificación del uso de las matemáticas en la sociedad.

\section{El Modelo Espacio de Trabajo Matemático}

El objetivo de ampliar el modelo introducido en Geometría por Kuzniak y Houdement (1999) al modelo teórico de los Espacios de Trabajo Matemático (ETM, ver KUZNIAK, 2006, 2011; KUZNIAK; RAUSCHER, 2011) es proporcionar una herramienta para el estudio específico del trabajo matemático de los estudiantes y profesores en el ámbito escolar. El espacio abstracto así concebido se refiere a una estructura organizada que permite observar las actividades de los individuos cuando se enfrentan a los problemas matemáticos. En el caso de las matemáticas escolares, estas personas generalmente no son expertos, son los estudiantes, experimentados o principiantes.

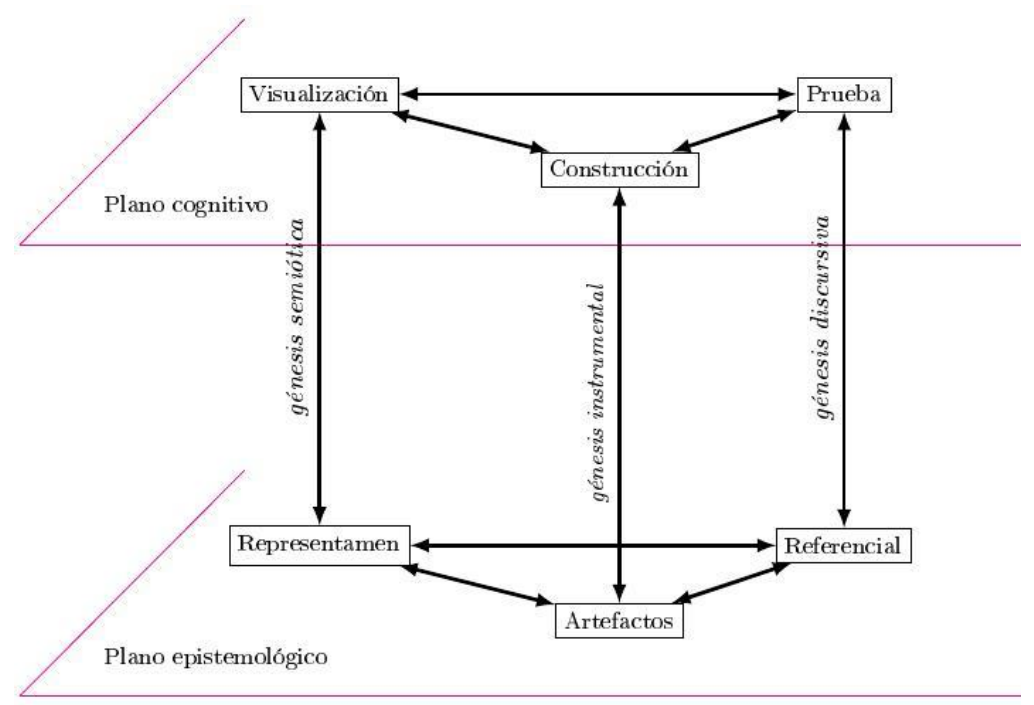

Figura 1 - Diagrama de Espacio de Trabajo Matemático

Del estudio inicial de este concepto en Geometría, conservamos el principio que articular el ETM en dos planos: uno de naturaleza epistemológica, en relación estrecha con los 
contenidos matemáticos del ámbito estudiado $\mathrm{y}$, el otro, de naturaleza cognitiva, que concierne al pensamiento del sujeto que resuelve tareas matemáticas (Fig. 1).

\subsection{Plano epistemológico y sus componentes}

Tres componentes en interacción son características de la actividad en su dimensión puramente matemática: un espacio real y local como soporte material, con un conjunto de objetos concretos y tangibles; un conjunto de artefactos como herramientas o software de dibujo o de cálculo; un sistema teórico de referencia basado en definiciones, teoremas, propiedades y axiomas.

Estas componentes no están yuxtapuestas, deben ser organizadas según un objetivo que depende del ámbito matemático en su dimensión epistemológica. Cuando el acento en una situación didáctica se pone en el proceso de aprendizaje del alumno este nivel epistemológico también se puede considerar como un medio epistemológico (COUTAT; RICHARD, 2011).

De acuerdo con una concepción de las matemáticas fundamentada en representaciones semióticas, que va más allá de la pura consideración de sistemas de representación, parece pertinente utilizar la noción de signo o representamen para resumir la componente relativa a los objetos tangibles y concretos. De acuerdo con Peirce (1931) el signo o representamen es algo que representa otra cosa, bien sea su objeto o quizá a él mismo. En función del ámbito matemático en cuestión, los signos podrán ser dibujos geométricos, símbolos algebraicos o gráficas, incluso fichas, maquetas o fotos, en el caso de problemas que abordan la modelización. Dependiendo del estudio, el conjunto de representamen puede organizarse en representaciones semióticas (DUVAL, 1995, 2006) o la interacción entre registros en haces semióticos (semiotic bundles) (ARZARELLO, 2006).

La noción de artefacto que utilizamos proviene de Rabardel (1995) e incluye todo lo que sufre una transformación, por pequeña que sea, de origen humano. Según Rabardel, su significado no se limita a los objetos materiales, sino que incluye los sistemas simbólicos. En el marco de los ETM, el significado de artefactos sigue siendo relativamente limitado para evitar la confusión con las otras componentes del plano epistemológico. Además, de los artefactos materiales tradicionales (como la regla, el compás o una calculadora) y de software informáticos (Software de Geometría Dinámica, hojas de cálculo, etc.), consideramos también como artefactos simbólicos los algoritmos y rutinas (cálculos técnicos planteados por 
números, las resoluciones de las ecuaciones de primer o segundo grado, derivación de polinomios, ...), los cuales están estrecha vinculación con la persona, como quedará explicitado en la descripción de este plano.

En contraste con representamen y artefactos, el conjunto de propiedades, teoremas, definiciones y axiomas se refiere a la parte teórica de trabajo matemático y es por eso que lo denominamos referencial teórico. Esto no es sólo una colección de propiedades, sino que sostiene el discurso deductivo de la demostración matemática, lo que implica que se organice de forma coherente y adaptada a las tareas propuestas a los estudiantes.

\subsection{El plano cognitivo y sus componentes}

Como se formula anteriormente (sección 1), las matemáticas que se enseñan no son un corpus desprovisto de propiedades y objetos reducidos a significantes manipulables mediante sistemas formales. De entrada, las matemáticas son principalmente una actividad humana. De esta manera, es esencial comprender cómo comunidades de individuos, pero también individuos particulares, utilizan y se apropian de los conocimientos matemáticos en sus prácticas de la disciplina. Asimismo, es esencial comprender cómo se va a dar sentido a estos signos y objetos tangibles. Lo que implica un segundo plano del ETM, centrado en el sujeto, que a su vez, se contempla como sujeto cognitivo. Esta apertura hacia el campo cognitivo se debe realizar en estrecha relación con las componentes del nivel epistemológico para las que se precisan tres componentes cognitivas: la visualización relativa a la representación del espacio y al soporte material; la construcción que depende de los instrumentos y técnicas asociadas y la demostración apoyada en el proceso discursivo de validación, basados en el referencial teórico.

El proceso de visualización se necesita precisar en este amplio contexto. Se debe asociar a esquemas y operaciones de uso de los signos, de los que nada nos prueba que dependan, a priori, de la visualización en sí. Este proceso de visualización extendida puede concebirse como un proceso de estructuración de la información proporcionada por los diagramas y los signos. Tiene que ser así distinguida de la simple visión o la percepción de los objetos.

\subsection{Dimensiones semiótica, instrumental, discursiva y sus génesis}


El desarrollo de su trabajo matemático por comunidades o individuos se lleva a cabo gradualmente y pasa por el establecimiento progresivo de su ETM personal. Esta génesis global del ETM supone un conjunto de génesis que son interdependientes y que involucran los planos epistemológico y cognitivo. Tres génesis se han identificado en este modelo: semiótica, instrumental y discursiva.

La génesis semiótica es el proceso asociado con signos y representamen que permite pasar de una perspectiva sintáctica a una perspectiva semántica de objetos matemáticos organizada en la representación semiótica. La génesis semiótica proporciona el estado de los objetos tangibles y sus operaciones. De esta manera, se asegura las relaciones entre la función y estructura en los signos.

Duval (2005), en la Geometría, menciona dos niveles de las operaciones correspondientes a este proceso. El primero de ellos es icónico y se asocia con el reconocimiento de percepción de formas, mientras que el segundo está relacionado con una interpretación más abstracta de los signos, con la idea principal de figura como un objeto simbólico. En nuestro estudio vamos a reconocer el mismo tipo de acción en otras disciplinas -en el Álgebra (en la expresión algebraica), en el Cálculo con diferentes perspectivas en gráficos y curvas-. Este tipo de transición identificada es una forma de transición entre los niveles icónicos, indexales y simbólico que encontramos en la semiótica.

La génesis instrumental permite hacer los artefactos operativos en el proceso de construcción en el Espacio de Trabajo Matemático. Seguimos la distinción realizada por Rabardel (1995) al considerar como un instrumento dos componentes: un artefacto, material o simbólica, y los esquemas de uso asociados. En esta perspectiva, la palabra instrumento tiene un valor cognoscitivo: un artefacto se convierte en un instrumento cuando el sujeto construye una serie de esquemas para su uso. En el modelo ETM, usamos la palabra herramienta para referirnos a las componentes del plano epistemológico, cuyo potencial de uso en la resolución de un problema generaliza la noción de instrumento de Rabardel. Los ordenadores han revolucionado completamente la cuestión del papel de los instrumentos en matemáticas, facilitando la posibilidad de desarrollar demostraciones dinámicas. Artigue (2002) destaca la necesidad de una génesis instrumental en dos direcciones principales, las cuales han sido consideradas en nuestro modelo. Un proceso hacia arriba, de los artefactos a la construcción, como configuraciones geométricas o cálculo, denominada instrumentación, describe la manipulación de los usuarios y el dominio de las herramientas. El proceso hacia abajo, desde la configuración a la elección adecuada de la herramienta, se llama instrumentalización. En 
este segundo proceso el conocimiento matemático se desarrolla.

La génesis discursiva de la prueba matemática utiliza las propiedades del sistema de referencia teórico para ponerlas al servicio del razonamiento matemático y para una validación no solamente icónica, gráfica o instrumental. En la génesis discursiva de la prueba, las propiedades utilizadas en el razonamiento matemático dan el significado. El propósito de esta génesis es proceder a la validación del proceso bidireccional: un razonamiento discursivo apoyado en las propiedades del referencial teórico y de otra, la identificación de propiedades y definiciones que se deben incluir en el marco de referencia después de ser realizado un tratamiento instrumental o semiótico.

La génesis discursiva de la prueba no agota todo lo relativo a las operaciones discursivas en matemáticas, donde es necesario distinguir tres niveles: la denominación de los objetos, el enunciado de propiedades y, finalmente, la deducción. La definición o la designación de los objetos es más una cuestión de registro del lenguaje y entra más en la dimensión puramente semiótica del trabajo matemático.

\subsection{Los planos verticales y la circulación en el ETM}

Como vimos, los planos epistemológico y cognitivo estructuran los ETM ayudan a comprender la circulación de los conocimientos en el seno del trabajo matemático. ¿Cómo articular de manera operatoria los niveles epistemológicos y cognitivos con el fin de hacer posible el trabajo matemático esperado? ¿Cómo organizar y describir la relación existente o formada entre las génesis?

A fin de definir el trabajo geométrico en el marco de los ETM de la geometría $\left(\mathrm{ETM}_{\mathrm{G}}\right)$, Coutat y Richard (2011) describen las interacciones específicas del enfoque geométrico caracterizando los tres planos verticales que aparecen en el diagrama de los ETM $_{\mathrm{G}}$. Los planos verticales introducidos, de este modo, se podrían conectar con las diferentes fases del trabajo matemático implementado en la ejecución de una tarea: descubrimiento y exploración, justificación y razonamiento, presentación y comunicación. De hecho, la ejecución efectiva de estas fases definirá un cierto número de competencias matemáticas cognitivas fundamentadas en la coordinación de las génesis en sus relaciones con el plano epistemológico (Fig. 2).

Basado en el estudio de Gómez-Chacón y Kuzniak (2015) presentamos estos planos con objeto de avanzar en el conocimiento de ETM.

El plano [Sem-Dis] es asociado a las génesis semiótica y discursiva de la prueba 
matemática. Una estrecha relación entre la génesis semiótica y la génesis discursiva de la prueba es crucial en el desarrollo del trabajo matemático que supera una visión simple icónica de los objetos. De acuerdo a la prioridad dada a la dimensión semiótica y discursiva, son posibles dos tipos de enfoques. Cuando la atención se centra en el lado semiótico, transformaciones visuales estructuran la descripción de los signos y organizan un razonamiento perceptivo. Por el contrario, si la atención se centra en una prueba o demostración el razonamiento hipotético y deductivo se basa en propiedades, signos y la visualización desempeña un papel heurístico.

El plano [Ins-Dis] es asociado a la génesis discursiva de prueba y a la génesis instrumental. En este plano, el punto crucial es la cuestión de la prueba que se basa en experimentos o en la argumentación deductiva pura. Si se sacan conclusiones a partir de datos dados por instrumentos, vamos a hablar de una prueba experimental. De otra forma, si la prueba o demostración se basará en un referencial teórico, los instrumentos se utilizan para ilustrar o para la construcción de configuraciones geométricas.

El plano [Sem-Ins] es asociado a una génesis semiótica y a la génesis instrumental. Este plano ha recibido más importancia debido a la aparición de Software Digital. Las herramientas digitales aumentan la capacidad de explorar configuraciones y descubrir nuevas propiedades. El enfoque exploratorio ya existía, pero sólo con base en la experiencia de los estudiantes. En este caso también, se pueden observar dos formas de trabajar: la que está más orientado hacia la construcción de los resultados (figuras, gráficos) que cumplen algunas condiciones y otra hacia la interpretación de los datos aportados por los artefactos.

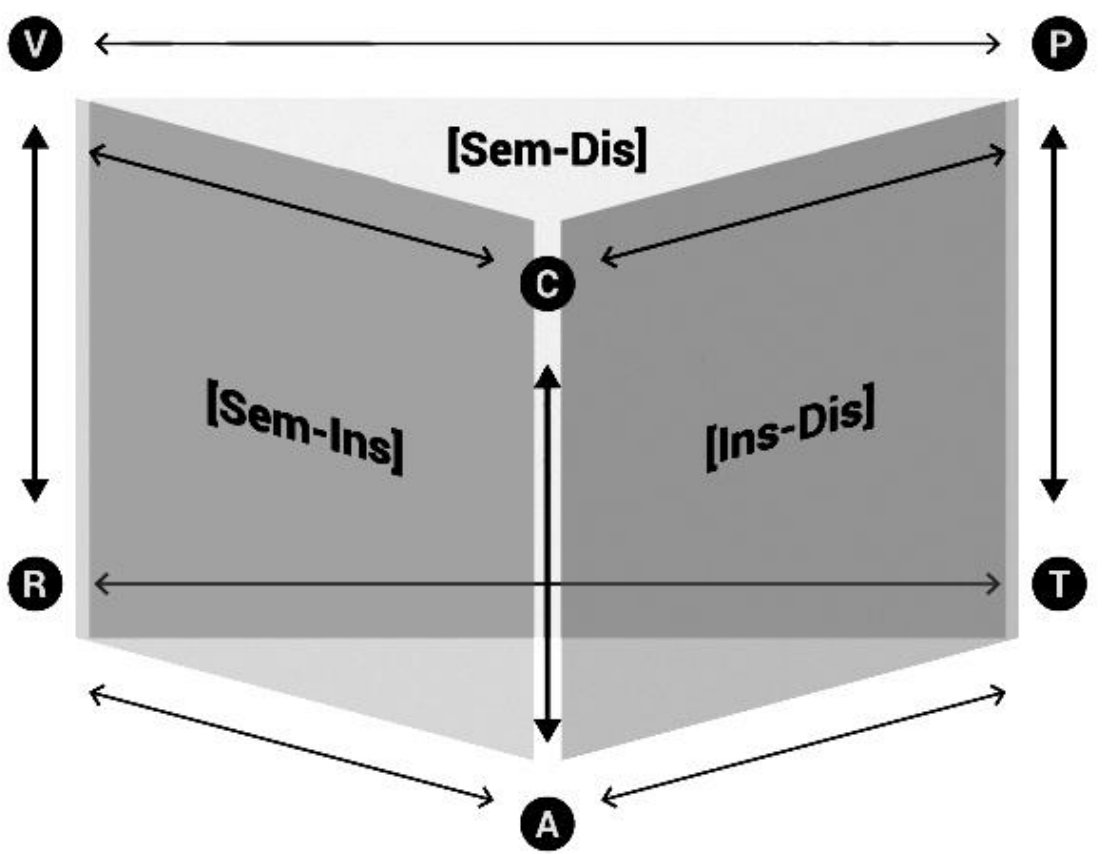

Figura 2 - Planos verticales en el ETM (extracto de la afiche del congreso ETM 5) 
La definición exacta de estos planos y la descripción de sus interrelaciones depende del dominio matemático específico que se estudia y permanece como un punto central en el estudio del trabajo matemático a través del modelo de ETM. El término fibración (RECIO; RICHARD; VIVIER, 2015) se sugirió para etiquetar movimientos y transiciones entre los diferentes elementos del modelo. De esta manera, tendríamos fibraciones internas (entre planos, entre las componentes, entre registros semióticos de representación...) y las fibraciones externas entre ETM asociados con diferentes dominios de matemáticas (MONTOYA DELGADILLO; VIVIER, 2014). Estas fibraciones visibilizan estas interdependencias y favorecen la descripción del edificio y la circulación del trabajo matemático en el modelo. Así mismo, favorece la deconstrucción de todos estos vínculos cuando las componentes se muestran fusionadas en el pensamiento confuso de los alumnos.

\subsection{Los tres tipologías de ETM: de referencia, idóneo y personal}

En esta sección, volvemos más específicamente a la cuestión del desarrollo del trabajo matemático en el ambiente escolar. En este caso, el camino que lleva a los estudiantes a la realización coherente de este trabajo marcado en primer lugar por las instituciones educativas que establece la dirección y, también por los docentes, quienes deben supervisar este trabajo en su clase diaria. Esto nos lleva a introducir tres tipos de ETM: ETM de referencia, ETM idóneo y ETM personal.

En el marco teórico de los ETM la noción de paradigma orienta y estructura la organización de las componentes que, debido a sus funciones diferentes, participan en la especificidad de los diversos paradigmas en juego. Un paradigma se instituye cuando una comunidad de individuos acuerda formular problemas, así como organizar sus soluciones, privilegiando ciertas herramientas o ciertas formas de pensamiento. Al espacio de trabajo paradigmático, tal como es definido por esta comunidad, se le llamará ETM de referencia. En una institución escolar dada, la resolución de un problema supone que un ETM idóneo se puede organizar para permitir a un alumno comprometerse en la resolución del problema. Este ETM idóneo debe necesariamente cumplir dos condiciones: por una parte, posibilitar el trabajo en el paradigma correspondiente a la problemática considerada; de otra parte, estar bien construido, en el sentido en que sus diferentes componentes están organizadas de manera válida. El diseñador desempeña un rol parecido al del arquitecto que diseña un espacio de trabajo para usuarios potenciales. En clase, el diseño de este espacio va a depender del ETM 
personal del profesor. Cuando el problema se propone a un alumno, el tratamiento matemático que éste le da le conduce al ETM personal de trabajo. Debido a esto, el ETM idóneo no es fijo y se debe modificar continuamente para ajustarse a las restricciones locales.

De esta manera, el trabajo matemático en un marco escolar se puede describir gracias a estos tres niveles de ETM: la matemática considerada por la institución que se describe en el ETM de referencia, éste es desarrollado por el profesor hasta alcanzar un ETM idóneo que permita un establecimiento efectivo en clase, donde cada alumno trabaja en su ETM personal.

La elección y la organización de las tareas propuestas a los alumnos por los profesores son esenciales en la constitución del ETM idóneo. Ofrece la posibilidad de resolver, de manera adecuada, lo que se les propone; es decir, conforme a las expectativas institucionales descritas de manera más o menos explícita en el ETM de referencia. Estas elecciones y la gestión de las actividades van a depender, en gran parte, del ETM personal del profesor. La observación de la actividad de los alumnos permitirá identificar sus ETM personales identificando posibles subconjuntos de prácticas estables.

\section{El trabajo matemático: el papel del profesor, sus conocimientos e interacciones}

La consideración del tema central de este monográfico parte de las discusiones en los Simposia ETM3, en Quebec, y ETM4, en España, sobre profundizar en el trabajo matemático del profesor en el aula y en la formación. En ambos Simposia el tema 3 se ha centrado en el rol de los docentes y las interacciones en la creación de un ETM idóneo y de ETMs personales eficientes. En la clase, el profesor ajusta y equilibra la dinámica del ETM. Se trata de dar respuesta a cómo gestionar las interacciones del trabajo matemático en el aula. Es un reto integrar de forma holística distintas dimensiones: cognitiva, didáctica, técnica, afectiva y cultural, en el análisis de estas interacciones y en la construcción del pensamiento matemático. En este monográfico Boletim de Educação Matemática (BOLEMA) de forma específica se reflexiona en las dinámicas que establecen en el rol del profesor, los conocimientos y las interacciones en el aula tanto con estudiantes como con los instrumentos. La monografía se ha estructurado en dos partes:

1. La formación de profesores;

2. El trabajo del profesor con los alumnos.

Al respecto de la utilización del modelo de los ETM, cada parte comienza con artículos basados principalmente en el modelo de los Espacios de Trabajo Matemático. Por 
esta razón, además de este artículo que ofrece un fundamento teórico del mismo, se incluyen cuatro artículos con objeto de profundizar en algunos elementos esenciales del modelo. Las otras contribuciones al tema de este monográfico no sólo han tenido como modelo teórico ETM, sino que han utilizado otros modelos teóricos de comprensión del conocimiento del profesor y del rol del profesor en el aula. En estos artículos se articulan el modelo de los ETM con otro marco teórico poniendo de manifiesto la eficacia de esta combinación o sinergia para la investigación desarrollada.

\subsection{La formación de profesores}

El primero de los artículos que inicia el tema de la formación es el de Nikolantonakis y Vivier. Se aborda el ETM en los sistemas numéricos posicionales en un estudio comparado con futuros profesores de primaria en Grecia y en Francia, mostrando que los ETM personales e idóneos son guiados por el sistema de numeración en base diez. Tratan de dar respuesta de cómo construir un $\mathrm{ETM}_{\mathbf{N}}$ idóneo para integrar las numeraciones en otras bases diferentes de la de diez profundizando en la génesis semiótica.

El secundo artículo, de Henríquez-Rivas y Montoya-Delgadillo, se centrará en temáticas de geometría euclidiana que articulan los enfoques sintético y analítico en formación de profesores de Secundaria, presentando una situación de referencia que favorece rutas de trabajo específicas. Los análisis se focalizan sobre el ETM personal de un futuro profesor y las transiciones entre los diferentes paradigmas de la geometría.

Gómez-Chacón, Botana, Escribano y Abánades proponen algunos elementos para organizar un espacio de trabajo efectivo para problemas de lugares geométricos en entornos tecnológicos. Se explora cómo futuros profesores de matemáticas progresan en su concepción de lugares geométricos a través de la apropiación de las funcionalidades específicas de cada entorno (tres herramientas), en relación con su propia práctica como estudiantes y su futuro ejercicio profesional. Las funcionalidades didácticas proporcionadas desde el diseñador se han estudiado desde el modelo Espacio de Trabajo Matemático (ETM). Este modelo pone de relieve la necesidad de articular para el trabajo geométrico los niveles epistemológico y cognitivo a través de diferentes génesis de razonamiento (visual-discursiva, instrumental y discursiva).

Bajo un enfoque de metodología observacional y de trabajo colaborativo Codina $\mathbf{y}$ Romero, en su artículo Entornos Tecnológicos y su Influencia en los Espacios de Trabajo 
Matemático, analizan la influencia de contextos ricos en tecnología digital sobre los Espacios de Trabajo Matemático (ETM). En particular, se describe cómo el uso de la tecnología propicia, de una parte, la modificación de roles en los agentes implicados en el proceso de enseñanza-aprendizaje y, por otra, la aparición de ETM compartidos.

Flores y Pluvinage exploran en la formación docente las Génesis Semiótica de los números Enteros desde una perspectiva didáctica e histórica. Muestran la riqueza y el interés de la construcción de Decartes para el producto de dos enteros.

Seguidamente, el artículo de Rodd reflexiona, desde un marco práctico que tiene en cuenta el modelo de ETM y las perspectivas de neurociencia, sobre la intervención del profesor en aspectos cognitivos y afectivos en las transiciones del razonamiento deductivo en la Geometría Euclídea.

Finalmente, esta parte concluye con el estudio de Cyrino. Su artículo está basado en algunos resultados interesantes del Grupo de Estudios e Investigación sobre Formación de Profesores para enseñar Matemáticas - GEPEFOPEM (Brasil). Abordan el tema de identidad profesional en relación al razonamiento proporcional en una comunidad de práctica, aportado un enfoque metodológico para precisar relaciones entre identidad y conocimiento matemático.

\subsection{El trabajo del profesor con los alumnos}

Montoya-Delgadillo, Mena-Lorca y Mena-Lorca, desde un contexto chileno, priorizarán el plano epistemológico en su trabajo con profesores debutantes de Secundaria. Ante tareas concretas mostrarán las formas que estos profesores dan concreción a las génesis semiótica, instrumental y discursiva, y a la circulación entre los distintos polos de los planos epistemológico y cognitivo.

El modelo teórico Mathematics Teacher's Specialised Knowledge (MTSK) se ha utilizado para profundizar en el conocimiento de la estructura matemática y en el establecimiento de categorías del Pedagogical content knowledge. Este modelo se presenta en el artículo de Flores-Medrano, Montes, Carrillo, Contreras, Muñoz-Catalán y Liñán, poniendo de manifiesto que el profesor desempeña un papel crucial en la articulación de los Espacios de Trabajo Matemático. El modelo de conocimiento del profesor MTSK propone una estructuración de dicho conocimiento basada en la noción de especialización. Esta estructuración, particularmente la consideración de sus componentes (subdominios), permite comprender el conocimiento que el profesor pone en juego en la transición entre los distintos 
Espacios (idóneo, personal y de referencia) de Trabajo Matemático, así como las relaciones entre estos.

Con base en los dos modelos teóricos: el modelo Mathematics Teacher's Specialised Knowledge (MTSK) y los Espacios de Trabajo Matemático (ETM), los autores Vasco, Climent, Escudero-Ávila, Montes y Ribeiro discuten sobre el conocimiento especializado (MTSK) que evidencia en una sesión sobre la multiplicación de matrices un profesor universitario de álgebra lineal y el Espacio de Trabajo Matemático (ETM) idóneo del profesor en esta sesión.

Con fundamentación en la Teoría de la Actividad y en el método de enseñanza de aprendizaje en colaboración, debate científico y autorreflexión (ACODESA), los autores Cortés, Hitt y Saboya nos ofrecen los resultados de dos experimentaciones en Canadá y en México. Abordan el problema de la enseñanza del Álgebra bajo el punto de vista del marco teórico de los Espacios de Trabajo Matemático (ETM). La interpretación descrita se centra en el paso de un plano epistemológico hacia un plano cognitivo en donde la actividad matemática permite una articulación de procesos de visualización, producción y procesos de validación aritmético-algebraicos que proporcionan un soporte precisamente al pensamiento aritmético-algebraico.

También, esta segunda parte del monográfico se profundiza en algunos aspectos específicos de intervención del profesor. El artículo de Panero, Arzarello, Sabena, con profesores italianos, se centra en la noción de derivada, analizando el proceso de generación de ejemplos y los procesos de visualización y semiótica.

Finalmente, encontramos el artículo de Gagatsis, Deliyianni, Elia, Panaoura y Michael-Chrysanthou que se centra en el nivel cognitivo del Espacio de Trabajo Matemático (ETM) y el componente del nivel epistemológico relacionado con las representaciones semióticas en dos dominios matemáticos de números racionales: fracción y número decimal. Dentro de este ámbito, se pretende explorar cómo la flexibilidad representacional se desarrolla con el tiempo. Se identifica un patrón de desarrollo similar de cuatro niveles jerárquicos de flexibilidad en ambos dominios y se discuten las implicaciones didácticas con el fin de fomentar la flexibilidad de representación en los ETM de fracción y la adición decimal.

\section{Primeras conclusiones y cuestiones abiertas para futuras investigaciones}

En esta sección se pretende delinear algunas conclusiones. Debido a la riqueza y a la 
complejidad de las cuestiones tratadas en cada uno de los artículos no pretendemos ser exhaustivos. Explicitaremos dos bloques de conclusión que por su naturaleza interrelacionada puede abrir perspectivas de futuro:

- La interacción entre los planos epistemológicos y cognitivo;

- El análisis del trabajo matemático del profesor en el aula y elementos importante a tener en cuenta en su formación.

\subsection{La interacción de los planos epistemológico y cognitivo}

El modelo de Espacios de Trabajo Matemático, concebido como una circulación entre distintos polos en los planos epistemológico y cognitivo, se puede considerar una herramienta del análisis (a priori tanto como a posteriori). Una herramienta de tareas de interpretación y descripción que debe permitir la implementación de un ajuste fino de las tareas ya construidas pero también debe la elaboración y calibración de nuevas situaciones educativas, o de situaciones que aún deben ser experimentadas.

Respecto a esta línea de conclusión es de interés las conclusiones obtenidas por las investigaciones: a) en temáticas específicas como los espacios matemáticos numéricos y el sistema posicional o en temáticas de geometría euclidiana que articulan los enfoques sintético y analítico; b) en la caracterización de los espacios de trabajo personal respecto al uso tecnológico, que pone de manifiesto que la articulación semiótico-figural, instrumental y discursivo dependen del funcionamiento matemático de los sujetos (aspectos epistemológicos y formas de resolución de los problemas); y de los bloqueos o dificultades en relación a la transición entre génesis; c) en el entrelazado de las tres génesis: tipo de validaciones que da norma a la génesis discursiva de la demostración, la discursividad involucrada en la génesis semiótica, la complejidad generada por la articulación de las génesis con las herramientas matemáticas.

\subsection{El análisis del trabajo matemático del profesor en el aula y los elementos importante a tener en cuenta en su formación}

En este monográfico se considera la teoría del ETM como un modelo dinámico que permite reflexionar sobre la acción del profesor en el aula y sobre elementos importante a tener en cuenta en su formación. Los ETM se construyen con base en el conocimiento 
matemático y la experiencia del sujeto al hacer matemáticas, de ahí que su organización remita a dos planos: el epistemológico y el cognitivo, que serán el centro de la discusión de este trabajo.

Se ha presentado el modelo a través de distintos estudios realizados en distintos países, proporcionando ejemplos de cómo puede actuar como una herramienta para el estudio de un trabajo efectivo de los profesores.

Inicialmente este modelo propuesto por Houdement y Kuzniak no se pensó como herramienta para la formación del profesorado, sin embargo, la perspectiva didáctica del trabajo matemático y el dinamismo de sus componentes ha posibilitado su uso como una herramienta para analizar cómo el profesor trabaja con las matemáticas. Estudiar el comportamiento del profesor no sólo como matemático -su ETM personal- sino también, permite verlo como profesor en la concepción de la matemática que debe enseñar, en el proceso de transposición de ésta con el objeto de elaborar propuestas didácticas que logren construcción de saberes matemáticos, y todas las reorganizaciones que su rol de profesor supone, en el ETM idóneo.

En este monográfico, para la reflexión del rol del profesor en la articulación de los Espacios de Trabajo Matemático para el aula se ha requerido del análisis del conocimiento del profesor, para ello se intentó ver qué articulación se podría producir entre el modelo de ETM y el modelo Mathematics Teacher's Specialised Knowledge, y entre los Espacios de Trabajo de Referencia, Idóneo y Personales.

Algunas ideas producidas no sólo en este monográfico sino en la discusión iniciada en el Simposium ETM4 (GÓMEZ-CHACÓN; ROMERO; CARRILLO, 2015) se pueden explicitar de la forma siguiente. El modelo de ETM (Fig. 1) planteado por Kuzniak (2011), donde se pone de manifiesto la articulación entre el plano epistemológico (la actividad en su dimensión puramente matemática) y el plano cognitivo con sus tres procesos cognitivos implicados en la actividad matemática requeriría una mayor especificación para episodios de clase. Una articulación que se podría producir en un análisis de episodio de clase entre el modelo de ETM y el modelo Mathematics Teacher's Specialised Knowledge podría ser expresada a través de la Figura 3. 


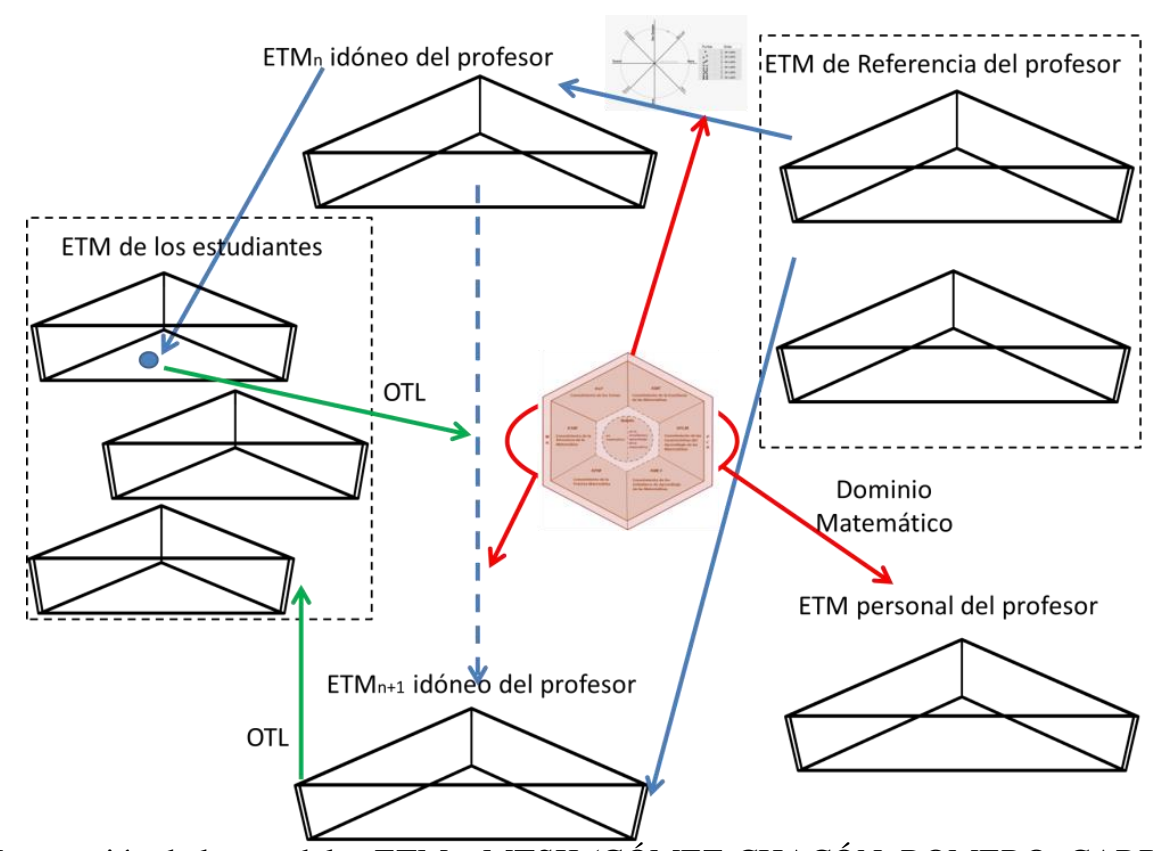

Figura 3 - Integración de los modelos ETM y MTSK (GÓMEZ-CHACÓN; ROMERO; CARRILLO, 2015)

Hacemos notar que se ha planteado una transformación del diagrama de la Figura 1, tomando la imagen de prismas (Figura 3) para mostrar la concepción de que la epistemología y los planos cognitivos son una herramienta analítica de procesos del trabajo matemático que actúan de manera integrada.

Partimos de una situación didáctica que el profesor propone a sus alumnos en el entorno del aula. En este entorno, el ETM Personal del estudiante es parte de una compleja red compuesta por el ETM Personal de los miembros del aula, incluyendo a sus compañeros y al profesor. La situación inicial se puede ver como una oportunidad de aprendizaje que influye en el ETM Personal de cada estudiante de un cierto modo. Los estudiantes tienen ETM Personales con diferencias y puntos en común. El hecho de que compartan características comunes permite al profesor planificar para el conjunto, así como valorar en conjunto el resultado de una intervención. El hecho de que existan diferencias entre los sujetos implica la posibilidad de enriquecer los ETMs Personales de distintos modos. Por ejemplo, el profesor puede observar el bloqueo en el trabajo de algún estudiante y pedir a otro que no lo tenga que hable sobre ello; o él puede ver un buen potencial de génesis de un estudiante y pedirle que lo comparta con el resto. Además, los estudiantes que trabajan de forma colaborativa en parejas o en entornos virtuales influyen en el ETM Personal de sus compañeros.

Así, desde la situación didáctica anterior, surgen significativas oportunidades de aprendizaje mientras los estudiantes trabajan sobre ella en el aula. Es trabajo del profesor detectar estas oportunidades y conducirlas de manera productiva. Para ello, el profesor debe adaptar el ETM idóneo a las diferentes oportunidades cuando surgen (por ejemplo, introducir 
un nuevo recurso para salir de un bloqueo o un nuevo concepto para nombrar una idea que surge en el grupo). Para este propósito, el modelo ETM se presenta adecuado y presenta una naturaleza dinámica sobre la que debe reflejarse, no sólo al principio y final de la situación didáctica, sino también en la oportunidad particular que esta puede brindar en el entorno dado.

En la adaptación del ETM de Referencia al ETM Idóneo (en cada paso) el profesor pone en juego su conocimiento. Uno puede buscar este conocimiento usando el modelo MTSK (conocimiento especializado del profesor de matemáticas). Hay claramente una relación sólida entre el ETM Personal del profesor y su conocimiento matemático (MK, que es uno de los dominios de MTSK).

En síntesis, esta discusión ha puesto de manifiesto análisis y elementos a tener en cuenta en el rol del profesor en el diseño y gestión del ETM idóneo de la clase. El conocimiento del profesor desempeña un papel central en dichas tareas, de modo que modelos analíticos que pongan el foco en la especificidad de la matemática nos ayudarán a comprender cómo se configura dicho ETM idóneo. En el análisis de los episodios discutidos en la parte segunda de esta monografía, el modelo MTSK ha servido como una herramienta para comprender el conocimiento del profesor que sustenta sus acciones y explicar en gran parte acciones que conlleva el establecimiento del ETM idóneo del profesor. Consideramos que, ambos modelos, ETM y MTSK, comparten elementos que los hacen complementarios para analizar la actividad educativa en el aula.

Quedan abiertas numerosas cuestiones para futuras investigaciones relativas a la profundización en el papel que los subdominios del MTSK idóneo del profesor tienen en la articulación entre los planos cognitivos y epistemológicos de los alumnos e indagar en el potencial comprensivo que la articulación de ambos modelos posee para la comprensión del proceso de enseñanza y aprendizaje.

\section{Referencias}

ARTIGUE, M. Learning mathematics in a CAS environment: the genesis of a reflection about instrumentation and dialectics between technical and conceptual work. International Journal of computer for Mathematical Learning, Vergag, v. 7, n. 3, p. 245- 274, oct. 2002.

ARZARELLO, F. Semiosis as a multimodal process. Revista latinoamericana de investigación en matemática educativa, México, v. 9, n. 4, p. 267-299, dic. 2006.

BROUSSEAU, G. Cadres, jeux de cadres et théories des situations. In: La journée en hommage à Régine Douady, 2002, Paris. Actes..., Paris: Université Paris-Diderot, 2002, p. 73-82.

COUTAT, S.; RICHARD, P. R. Les figures dynamiques dans un espace de travail mathématique pour l'apprentissage des propriétés géométriques. Annales de didactique et de sciences cognitives, 
Strasbourg, v. 16, p. 97-126, 2011.

DUVAL, R. A cognitive analysis of problems of comprehension in a learning of mathematics, Educational Studies in Mathematics, Netherlands, v. 61, n. 1-2, p. 103-131, feb. 2006.

DUVAL, R. Les conditions cognitives de l'apprentissage de la géométrie. Annales de Didactique et de sciences cognitives, Strasbourg, v. 10, p. 5-54, 2005.

DUVAL, R. Sémiosis et pensée humaine: registres sémiotiques et apprentissages intellectuels. Berne: Peter Lang, 1995.

FREUDENTHAL, H. Geometry between the devil and the deep sea. Educational Studies in Mathematics, Netherlands, v. 3, nº3-4, p. 413-435, june. 1971.

GILLIES, D. Revolutions in Mathematics. Oxford Science Publication, Oxford:The Clarendon Press, 1992.

GÓMEZ-CHACÓN, I. M.; KUZNIAK, A. Geometric Work Spaces: figural, instrumental and discursive geneses of reasoning in a technological environment. International Journal of Science and Mathematics Education, Singapore, v. 13, n. 1, p. 201-226, feb. 2015.

GÓMEZ-CHACÓN, I. M. ROMERO I. M.; CARRILLO, J. Genesis and development of mathematical work: the role of the teacher and the interactions. In: GÓMEZ-CHACÓN, I. M. et. al (Ed.). Mathematical Working Space, Proceedings Fourth ETM Symposium. Madrid:

Publicaciones del Instituto de Matemática Interdisciplinar, Universidad Complutense de Madrid, 2015, p. $405-410$.

HACKING, I. Why is there philosophy of mathematics at all ? Cambridge: Cambridge University Press, 2014.

HOUDEMENT, C.; KUZNIAK, A. Un exemple de cadre conceptuel pour de l'enseignement de la géométrie en formation des maîtres. Educational Studies in Mathematics, Netherlands , v. 40, n. 3, p. 283-312, nov. 1999.

HOUDEMENT C. ; KUZNIAK A. Paradigmes géométriques et enseignement de la géométrie. Annales de Didactique et de Sciences Cognitives, Strasbourg, v. 11, p. 175 - 193, 2006.

KUHN, T.S. The structure of scientific revolutions, 2ed. Chicago: University of Chicago Press, 1966.

KUZNIAK, A. L'espace de Travail Mathématique et ses genèses. Annales de didactique et de sciences cognitives, Strasbourg, v. 16, p. 9-24, 2011.

KUZNIAK, A. Paradigmes et espaces de travail géométriques. Éléments d'un cadre théorique pour l'enseignement et la formation des enseignants en géométrie. Canadian Journal of Science and Mathematics Education, Toronto, v. 6 n. 2. p 167-188, jan. 2006.

KUZNIAK, A.; RAUSCHER, J. C. How do teachers' approaches on geometrical work relate to geometry students learning difficulties? Educational Studies in Mathematics, Netherlands, v. 77, n. 1, p. 129-147, may, 2011.

LEGRAND, M. Rationalité et démonstration mathématiques, le rapport de la classe à une communauté scientifique. Recherches en didactique des mathématiques, v. 9 n. 3, p. 365-406, 1990. 
MONTOYA-DELGADILLO, E.; VIVIER, L. Les changements de domaine de travail dans le cadre des Espaces de Travail Mathématique. Annales de Didactique et de Sciences Cognitives, Strasbourg, v. 19, p. 73-101, 2014.

PEIRCE C.S The collected papers. Cambridge: Harvard University Press, 1931. v.2.

RABARDEL, P. Les Hommes et les technologies: une approche cognitive des instruments contemporains. Paris, France: Armand Colin, 1995.

RECIO, T.; RICHARD, P. R.; VIVIER, L. Specific tools and signs in the mathematical work. In: GÓMEZ-CHACÓN, I. M. et. al (Ed.). Mathematical Working Space, Proceedings Fourth ETM Symposium. Madrid: Publicaciones del Instituto de Matemática Interdisciplinar, Universidad Complutense de Madrid, 2015. p. 207-216.

THURSTON, W. P. On proof and progress in mathematics. Bulletin of American Mathematical Society, Providence, v. 30, n. 2, p. 161-177, 1994.

VANDEBROUCK, F. (Ed.). Mathematics classrooms students' activities and teachers' practices.Rotterdam: Sense, 2013.

Submetido em Julho de 2015. Aprovado em Setembro de 2015. 